\title{
Antioxidant potential of microalgae in relation to their phenolic and carotenoid content
}

\author{
Koen Goiris • Koenraad Muylaert • Ilse Fraeye • \\ Imogen Foubert • Jos De Brabanter • Luc De Cooman
}

Received: 14 November 2011 / Revised and accepted: 24 January 2012

(C) Springer Science+Business Media B.V. 2012

\begin{abstract}
In the past decades, food scientists have been searching for natural alternatives to replace synthetic antioxidants. In order to evaluate the potential of microalgae as new source of safe antioxidants, 32 microalgal biomass samples were screened for their antioxidant capacity using three antioxidant assays, and both total phenolic content and carotenoid content were measured. Microalgae were extracted using a one-step extraction with ethanol/water, and alternatively, a three-step fractionation procedure using successively hexane, ethyl acetate, and water. Antioxidant activity of the extracts varied strongly between species and further depended on growth conditions and the solvent used
\end{abstract}

K. Goiris $\cdot$ L. De Cooman

KAHO Sint-Lieven, Laboratory of Enzyme, Fermentation,

and Brewing Technology,

Gebroeders De Smetstraat 1,

9000 Ghent, Belgium

K. Goiris $\cdot$ L. De Cooman

Leuven Food Science and Nutrition Research Centre (LFoRCe),

Department M2S, K. U. Leuven,

Kasteelpark Arenberg 20,

3001 Leuven, Belgium

K. Goiris $(\bowtie) \cdot K$. Muylaert

Research unit Aquatic Biology, K.U.Leuven Kulak,

Etienne Sabbelaan 53,

8500 Kortrijk, Belgium

e-mail: koen.goiris@kahosl.be

I. Fraeye $\cdot$ I. Foubert

Research unit Food and Lipids, K.U.Leuven Kulak,

Etienne Sabbelaan 53,

8500 Kortrijk, Belgium

J. De Brabanter

Departement Industrieel Ingenieur, KAHO Sint-Lieven,

Gebroeders De Smetstraat 1,

9000 Ghent, Belgium for extraction. It was found that industrially cultivated samples of Tetraselmis suecica, Botryococcus braunii, Neochloris oleoabundans, Isochrysis sp., Chlorella vulgaris, and Phaeodactylum tricornutum possessed the highest antioxidant capacities in this study and thus could be a potential new source of natural antioxidants. The results from the different types of extracts clearly indicated that next to the well-studied carotenoids, phenolic compounds also contribute significantly to the antioxidant capacity of microalgae.

Keywords Microalgae $\cdot$ Antioxidant capacity Phenolic content $\cdot$ Carotenoid content

\section{Introduction}

The use of antioxidants to prolong the shelf life of foodstuffs is ubiquitous. Today, mostly synthetic antioxidants, such as butylated hydroxytoluene (BHT) or butylated hydroxyanisole (BHA), are used. As these components are suspected carcinogens (Namiki 1990; Pokorný 1991), there has been a search in recent years to replace these synthetic antioxidants with natural antioxidants. The number of introductions of novel antioxidant formulations for use as food ingredient or as food supplements has been significant over the last two decades, and in many cases these novel antioxidants were of natural origin. Antioxidants are presumed to have several positive health effects, including prevention of cardiovascular disorders, of certain ageing related diseases such as Alzheimer and of certain types of cancer. Therefore, antioxidants are increasingly being used in food supplements and functional foods (Cuvelier 2001).

Most, if not all, commercially available natural antioxidants are derived from terrestrial plants (e.g. rosemary, tea, grape seeds, pine bark, cocoa). It is however believed that 
unicellular microalgae are a promising alternative source of antioxidants (Li et al. 2007; Natrah et al. 2007; Hajimahmoodi et al. 2010; Rodriguez-Garcia and Guil-guerrero 2008; Chacón-Lee and González-Mariño 2010; Lee et al. 2010). There are a number of reports on the evaluation of antioxidant activity in microalgae and cyanobacteria belonging to the genera Botryococcus (Rao et al. 2006), Chlorella (Wu et al. 2005; Goh et al. 2010), Dunaliella (Herrero et al. 2006), Nostoc (Li et al. 2007), Phaeodactylum (Guzman et al. 2001), Spirulina (Miranda et al. 1998; Jaime et al. 2005; Mendiola et al. 2007), Haematococcus (Cerón et al. 2007) and Chaetoceros (Goh et al. 2010). These studies concluded that several microalgal genera contain potent antioxidants, both from lipophilic and hydrophilic nature.

An important and well-known class of antioxidants from microalgae are carotenoids. Carotenoids play an important role in quenching reactive oxygen species (ROS) generated during photosynthesis, especially singlet oxygen. Several studies have demonstrated that carotenoids contribute significantly to the total antioxidant capacity of microalgae (Jahnke 1999; Takaichi 2011). Microalgae are already commercially produced as a source of carotenoid antioxidants (e.g. Haematococcus for astaxanthin, Dunaliella for $\beta$ carotene) for use as additives in food and feed applications, as well as for use in cosmetics and as food supplements (Pulz and Gross 2004; Spolaore et al. 2006).

In terrestrial plants, an important class of antioxidants are phenolic compounds, more specifically the relatively complex flavonoids, which show several antioxidant mechanisms. Flavonoids can inhibit lipid oxidation (Pietta 2000) by directly scavenging ${ }^{\bullet} \mathrm{OH}, \mathrm{HOCl}$, singlet oxygen and lipid peroxyl radicals, by metal chelation and by inhibiting lipoxygenase. Little information is however available about the presence of phenolic compounds in microalgae. Recent research by Klejdus et al. (2010) showed that several classes of flavonoids, such as isoflavones, flavanones, flavonols and dihydrochalcones can be found in microalgae and cyanobacteria. This indicates that, although microalgae are evolutionary more primitive than terrestrial plants, or even belong to completely different evolutionary lineages, they are capable of producing relatively complex polyphenols.

It is not clear whether phenolic substances are important antioxidants in microalgae. The fact that the content of phenolic substances in microalgal biomass increases upon exposure to UV-light (Duval et al. 2000; Kovácik et al. 2010) suggests that they indeed play a role in the antioxidative response to this type of stress. However, there is no consensus on the importance of phenolic constituents for the antioxidant capacity of microalgae. Several studies have compared antioxidant activity and phenolic content in fractionated extracts of biomass from one or a few species of microalgae. Three studies found that fractions that were rich in phenolic compounds also had a high antioxidant capacity (Jaime et al. 2005; Geetha et al. 2010; Custódio et al. 2011), while another study found the opposite (Goh et al. 2010). Other recent work looked at a more extensive set of microalgae to study the relation between antioxidant activity and phenolic content of the extracts. Li et al. (2007) used only a single test (Trolox Equivalent Antioxidant Capacity, TEAC) to evaluate antioxidant activity and found no relation between phenolic content and antioxidant capacity. A more recent study used two antioxidant tests (DPPH-radical scavenging and Ferric Reducing Antioxidant Potential, FRAP) and a significant relation between antioxidant activities and phenolic content was noticed (Hajimahmoodi et al. 2010). However, none of these studies took into account the contribution of carotenoids to the total antioxidant activity.

In this study, we investigated the contribution of phenolic substances to antioxidant activity in a series of extracts from 32 algal biomass samples to evaluate whether microalgae may be a valuable source of phenolic antioxidants. To take into account different antioxidant mechanisms, we carried out three different antioxidant activity assays used in food research. These include TEAC, FRAP and AIOLA, the latter of which has to our knowledge not previously been applied to algal biomass extracts. In order to assess the contribution of phenolic substances to antioxidant activity independent of that of carotenoids, we also determined carotenoid content and used multiple regression to estimate and compare the contribution of both phenolic compounds and carotenoids to the measured antioxidant activities.

\section{Materials and methods}

A total of 32 biomass samples were used in this study, of which 14 samples of freeze-dried biomass from pure cultures were provided by commercial producers: Phaeodactylum tricornutum (Bacillariophyceae), Nannochloropsis sp. (Eustigmatophyceae), Isochrysis sp. (Prymnesiophyceae), Tetraselmis suecica (Chlorophyta), Chaetoceros calcitrans (Bacillariophyceae), Schizochytrium sp. (Labyrinthulomycetes), Chlorella sp. 1 and sp. 2 (Chlorophyta) by SBAE Industries (Sleidinge, Belgium) and Porphyridium cruentum (Rhodophyta), Botryococcus braunii, Tetraselmis sp., Nannochloropsis oculata, Isochryis sp. and Neochloris oleoabundans (Chlorophyta) by Necton S.A. (Olhão, Portugal).

Samples of freeze-dried biomass were further obtained from pure cultures of 5 species produced inhouse under controlled conditions in pilot-scale photobioreactors (Parachlorella kessleri SAG 27.87, Chlorella vulgaris SAG 211-11B, Scenedesmus obliquus CCAP 276/3A Haematococcus pluvialis SAG 192.80 (Chlorophyta) and P. tricornutum UGent Pt86 (Bacillariophyta). The algal biomass of these species was 
produced in-house using $130 \mathrm{~L}$ plexiglass tubular airlift reactors. Algae were cultured in Wright's Cryptophyte (WC) medium (Guillard and Lorenzen 1972) prepared with either deionised water for the freshwater species or with synthetic seawater $\left(30 \mathrm{~g} \mathrm{~L}^{-1}\right.$ Homarsel, Zoutman, Belgium) for the marine species. The medium was filter-sterilised before addition to the photobioreactors (0.2 $\mu \mathrm{m}$ PTFE filters). The reactors were illuminated continuously $\left(125 \mu \mathrm{mol}\right.$ photons $\mathrm{m}^{-2} \mathrm{~s}^{-1}$, Philips Cool White fluorescent tubes) and mixed with filter-sterilised air (flow rate: $25 \mathrm{~L} \mathrm{~min}^{-1}$ ). The culture was maintained at $\mathrm{pH} 8.5$ by automated addition of $\mathrm{CO}_{2}$ to the stream of air. The biomass was harvested at the end of the logarithmic phase by centrifugation and the wet biomass was immediately freeze-dried and stored frozen at $-20^{\circ} \mathrm{C}$.

To evaluate to what extent antioxidant activity might vary between different batches of the same species produced under slightly different conditions, replicate batches were produced for C. vulgaris (for batch 1, the WC-medium contained $5 \mathrm{x}$ higher nutrients concentrations) and for $P$. tricornutum ( 3 batches). The main difference was the timing of the harvesting. C. vulgaris batches \#1 and \#4 were harvested 9 days after inoculation; batches \#2 and \#3 after 8 days and batches $\# 5$ and $\# 6$ after 6 days cultivation. $P$. tricornutum biomass \#1 was harvested after 9 days cultivation, whereas biomass \#2 and \#3 were harvested after 8 days. For H. pluvialis, biomass was collected during the exponential (green stage) and stationary (red phase) growth stages as it is known that these differ strongly in carotenoid content.

In addition, samples of mixed algal cultures were obtained from commercial producers: 2 samples of mixed algae produced on wastewater by Maris Projects (Schijndel, The Netherlands) and 3 samples of mixed algae to be used as aquaculture feed from SBAE Industries.

\section{Extraction of antioxidants}

Extracts from the biomass samples were obtained using two solid-liquid extraction procedures. Extractions were performed in the dark at room temperature under inert nitrogen atmosphere.

The first procedure aimed to extract both apolar and polar compounds using an ethanol/water mixture. For this, $200 \mathrm{mg}$ of freeze-dried biomass was ground using a pestle and mortar and extracted with $2 \mathrm{~mL}$ ethanol/water $(3 / 1 \mathrm{v} / \mathrm{v})$ mixture for 30 minutes. After centrifugation $(4500 \times \mathrm{g}$, $10 \mathrm{~min}$ ), the pellet was resuspended in $2 \mathrm{~mL}$ of the ethanol/water mixture and extracted for a second time. Both extracts were pooled and stored under nitrogen atmosphere at $-20^{\circ} \mathrm{C}$ prior to analysis.

The second procedure aimed to separate polar from apolar compounds using a sequential extraction in hexane, ethyl acetate and hot water ( $\mathrm{Li}$ et al. 2007). For this, $200 \mathrm{mg}$ freeze-dried biomass was ground in a mortar and extracted with $2 \mathrm{~mL}$ of hexane for $30 \mathrm{~min}$. After centrifugation, the pellet was resuspended in hexane and extracted for a second time, and both extracts were combined. The biomass pellet was subsequently extracted with ethyl acetate using the same procedure and finally with hot deionised water $\left(80^{\circ} \mathrm{C}\right)$. The hexane and ethyl acetate extracts were dried under a stream of nitrogen and redissolved in $1 \mathrm{~mL}$ ethanol. All extracts were stored under nitrogen atmosphere at $-20^{\circ} \mathrm{C}$ prior to analysis.

\section{Antioxidant measurements}

Antioxidant activity was measured by three assays commonly used in the food industry. In each assay, trolox was used as a reference.

The trolox equivalent antioxidant capacity or TEAC assay was carried out according to $\mathrm{Li}$ et al. (2007). In the TEAC assay, antioxidant action by hydrogen atom transfer (HAT) as well as single electron transfer (SET) is measured (Prior et al. 2005). For the assay, $\mathrm{ABTS}^{\bullet+}$ radical cation was generated by preparing a solution of $7 \mathrm{mM}$ ABTS and $2.45 \mathrm{mM}$ potassium persulphate in milliQ-water. The reaction mixture was allowed to stand in the dark for $16 \mathrm{~h}$ at room temperature and was used within two days. The $\mathrm{ABTS}^{\bullet+}$ solution was diluted with deionised water to give an absorbance of $0.700 \pm 0.050$ at $734 \mathrm{~nm}$. $50 \mu \mathrm{L}$ of sample was mixed with $1.9 \mathrm{~mL}$ of diluted $\mathrm{ABTS}^{\bullet+}$ solution. After 10 minutes incubation at room temperature, the absorbance was measured at $734 \mathrm{~nm}$.

The ferric reducing antioxidant potential or FRAP assay was carried out according to Hajimahmoodi et al. (2010). The FRAP-assay represents the antioxidant action via the SET-mechanism (Prior et al. 2005), but the test cannot

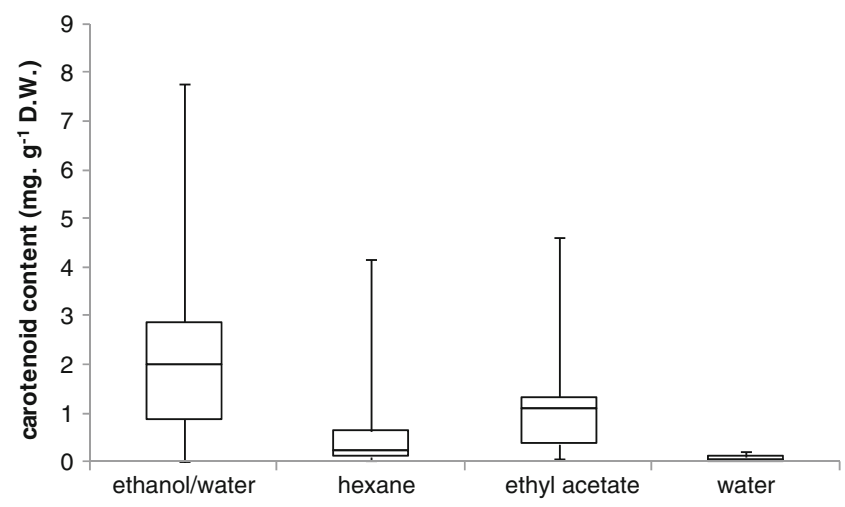

Fig. 1 Box plot distribution of the total carotenoid content of the microalgal fractions as calculated with the Lichtenthaler equations (Lichtenthaler and Buschmann 2001) using the absorbances at 470, 652 and $666 \mathrm{~nm}$. Error bars represent minimum and maximum values, whereas the rectangle boundaries represent 1 st quartile, median and 3th quartile values 
detect compounds that act by radical quenching. The FRAP reagent was freshly prepared by mixing equal volumes of a $10 \mathrm{mM}$ TPTZ (2,4,6-tripyridyl-S-triazine) solution in $40 \mathrm{mM} \mathrm{HCl}$ and a $20 \mathrm{mM} \mathrm{FeCl}_{3}$ (Merck, Germany) solution and diluting five times in a $0.3 \mathrm{M}$ acetate buffer ( $\mathrm{pH}$ 3.6) followed by warming to $37^{\circ} \mathrm{C} .100 \mu \mathrm{L}$ sample was mixed with $3 \mathrm{~mL}$ FRAP reagent and the absorbance of the reaction mixture was measured at $593 \mathrm{~nm}$ after incubating for 10 minutes at $37^{\circ} \mathrm{C}$. When necessary, extracts were appropriately diluted with ethanol.
The AAPH-induced oxidation of linoleic acid or AIOLA assay was described by Liégois et al. (2000) and measures the ability of an antioxidant to prevent oxidation of linoleic acid induced by alkylperoxyl radicals, generated from the water soluble azo compound 2,2'-azobis(2-amidinopropane) dihydrochloride. For the test, a linoleic acid emulsion was prepared by slowly adding $0.25 \mathrm{~mL}$ linoleic acid under continuous stirring to $5 \mathrm{~mL}$ of a $0.05 \mathrm{M}$ borate buffer (pH 9) containing $0.25 \mathrm{~mL}$ of Tween 20 . The resulting dispersion was clarified by adding $1 \mathrm{~mL}$ of $1 \mathrm{~N}$ sodium hydroxide. The

Table 1 Carotenoid content, phenolic content and antioxidant activity in ethanol/water extracts from microalgal biomass

\begin{tabular}{|c|c|c|c|c|c|}
\hline & $\begin{array}{l}\text { carotenoid content }^{\mathrm{a}} \\
\left(\mathrm{mg} \cdot \mathrm{g}^{-1} \text { D.W. }\right)\end{array}$ & $\begin{array}{l}\text { phenolic content }^{\mathrm{a}} \\
\left(\mathrm{mg} \text { GAE. } \mathrm{g}^{-1} \text { D.W. }\right)\end{array}$ & $\begin{array}{l}\text { TEAC }{ }^{\text {a }}(\mu \mathrm{mol} \\
\left.\text { trolox eq. } \mathrm{g}^{-1} \mathrm{D} . \mathrm{W} .\right)\end{array}$ & $\begin{array}{l}\text { FRAP }^{\text {a }}(\mu \mathrm{mol} \\
\left.\text { trolox eq. } \mathrm{g}^{-1} \text { D.W. }\right)\end{array}$ & $\begin{array}{l}\text { AIOLA }^{\text {a }}(\mu \mathrm{mol} \\
\left.\text { trolox eq. } \mathrm{g}^{-1} \text { D.W. }\right)\end{array}$ \\
\hline Botryococcus braunii & $2.10 \pm 0.07$ & $1.99 \pm 0.17$ & $53.90 \pm 0.76$ & $21.83 \pm 1.41$ & $10.42 \pm 0.63$ \\
\hline Chaetoceros calcitrans & $2.33 \pm 0.14$ & $1.84 \pm 0.11$ & $24.24 \pm 1.64$ & $4.74 \pm 0.89$ & $22.37 \pm 1.34$ \\
\hline Chlorella \#1 & $3.04 \pm 0.20$ & $2.21 \pm 0.26$ & $51.03 \pm 2.04$ & $6.37 \pm 0.24$ & $21.18 \pm 1.27$ \\
\hline Chlorella \#2 & $2.97 \pm 0.51$ & $1.47 \pm 0.07$ & $59.57 \pm 2.77$ & $9.32 \pm 0.51$ & $15.59 \pm 0.94$ \\
\hline Chlorella vulgaris batch $1 \mathrm{KAHO}$ & $1.93 \pm 0.19$ & $1.47 \pm 0.16$ & $19.97 \pm 1.48$ & $64.65 \pm 5.80$ & n.a. ${ }^{\mathrm{b}}$ \\
\hline Chlorella vulgaris batch $2 \mathrm{KAHO}$ & $1.02 \pm 0.09$ & $0.75 \pm 0.03$ & $9.33 \pm 0.24$ & $24.34 \pm 1.73$ & n.a. \\
\hline Chlorella vulgaris batch $3 \mathrm{KAHO}$ & $0.71 \pm 0.08$ & $1.30 \pm 0.25$ & $11.57 \pm 0.15$ & $42.00 \pm 2.40$ & n.a. \\
\hline Chlorella vulgaris batch $4 \mathrm{KAHO}$ & $0.71 \pm 0.03$ & $1.70 \pm 0.09$ & $10.40 \pm 0.15$ & $38.74 \pm 1.26$ & n.a. \\
\hline Chlorella vulgaris batch $5 \mathrm{KAHO}$ & $2.17 \pm 0.17$ & $1.59 \pm 0.22$ & $11.66 \pm 0.20$ & $37.05 \pm 0.25$ & n.a. \\
\hline Chlorella vulgaris batch $6 \mathrm{KAHO}$ & $0.25 \pm 0.09$ & $1.64 \pm 0.11$ & $5.40 \pm 0.12$ & $30.59 \pm 3.25$ & n.a. \\
\hline Haematococcus pluvialis 'red phase' & n.d. ${ }^{\mathrm{c}}$ & $0.54 \pm 0.05$ & $5.71 \pm 0.12$ & $17.36 \pm 0.58$ & n.a. \\
\hline Haematococcus pluvialis 'green phase' & $1.89 \pm 0.05$ & $1.23 \pm 0.06$ & $9.12 \pm 0.12$ & $41.34 \pm 2.93$ & n.a. \\
\hline Isochrysis ISO-T & $3.08 \pm 0.07$ & $2.67 \pm 0.22$ & $34.94 \pm 1.10$ & $46.69 \pm 0.48$ & $38.07 \pm 2.28$ \\
\hline Isochrysis sp. & $7.75 \pm 0.13$ & $4.57 \pm 0.18$ & $22.50 \pm 0.93$ & $53.73 \pm 3.21$ & $12.90 \pm 0.77$ \\
\hline Mixed culture \#1 (A.C. ${ }^{\mathrm{d}}$ ) & $0.50 \pm 0.02$ & $1.64 \pm 0.16$ & $20.10 \pm 1.69$ & $3.30 \pm 0.13$ & $14.92 \pm 0.89$ \\
\hline Mixed culture \#2 (A.C.) & $1.04 \pm 0.04$ & $1.26 \pm 0.06$ & $36.77 \pm 2.79$ & $5.31 \pm 0.71$ & $33.44 \pm 2.01$ \\
\hline Mixed culture \#3 (A.C.) & $2.08 \pm 0.05$ & $3.86 \pm 0.21$ & $34.50 \pm 2.12$ & $17.29 \pm 2.08$ & $45.30 \pm 2.71$ \\
\hline Mixed culture \#4 (W.W.T. ${ }^{\mathrm{e}}$ ) & $0.23 \pm 0.02$ & $1.69 \pm 0.02$ & $11.15 \pm 0.03$ & $7.93 \pm 0.54$ & $17.62 \pm 1.06$ \\
\hline Mixed culture \#5 (W.W.T.) & $1.39 \pm 0.02$ & $1.05 \pm 0.04$ & $20.75 \pm 1.59$ & $3.54 \pm 0.09$ & $7.82 \pm 0.47$ \\
\hline Nannochloropsis oculata & $1.65 \pm 0.10$ & $2.04 \pm 0.35$ & $20.16 \pm 0.98$ & $40.68 \pm 1.61$ & $6.47 \pm 0.40$ \\
\hline Nannochloropsis sp. & $2.17 \pm 0.03$ & $1.39 \pm 0.20$ & $25.83 \pm 1.09$ & $40.80 \pm 1.05$ & $28.81 \pm 1.73$ \\
\hline Neochloris oleoabundans & $1.56 \pm 0.29$ & $3.73 \pm 0.24$ & $64.30 \pm 4.57$ & $40.72 \pm 0.67$ & $16.56 \pm 0.99$ \\
\hline Parachlorella kessleri & $2.12 \pm 0.04$ & $1.38 \pm 0.16$ & $27.36 \pm 1.33$ & $39.36 \pm 1.77$ & $32.24 \pm 1.93$ \\
\hline Phaeodactylum tricornutum & $6.14 \pm 0.16$ & $3.75 \pm 0.46$ & $48.90 \pm 1.30$ & $89.70 \pm 1.43$ & $46.29 \pm 2.78$ \\
\hline $\begin{array}{l}\text { Phaeodactylum tricornutum } \\
\text { batch } 1 \text { KAHO }\end{array}$ & $2.88 \pm 0.04$ & $3.19 \pm 0.29$ & $11.88 \pm 0.29$ & $34.90 \pm 1.23$ & n.a. \\
\hline $\begin{array}{l}\text { Phaeodactylum tricornutum } \\
\text { batch } 2 \text { KAHO }\end{array}$ & $2.26 \pm 0.10$ & $3.54 \pm 0.29$ & $4.55 \pm 0.01$ & $37.19 \pm 0.83$ & n.a. \\
\hline $\begin{array}{l}\text { Phaeodactylum tricornutum } \\
\text { batch } 3 \text { KAHO }\end{array}$ & $3.14 \pm 0.06$ & $3.48 \pm 0.75$ & $11.07 \pm 0.19$ & $58.84 \pm 1.50$ & n.a. \\
\hline Porphyridium cruentum & $0.95 \pm 0.01$ & $1.22 \pm 0.05$ & $5.14 \pm 0.41$ & $10.89 \pm 0.17$ & $1.82 \pm 0.11$ \\
\hline Scenedesmus obliquus & $0.44 \pm 0.06$ & $1.94 \pm 0.16$ & $5.87 \pm 0.28$ & $19.68 \pm 1.52$ & n.a. \\
\hline Schizochytrium sp. & n.d. & $1.94 \pm 0.16$ & n.d. & $28.04 \pm 0.56$ & n.a. \\
\hline Tetraselmis sp. & $2.88 \pm 0.29$ & $3.74 \pm 0.10$ & $69.40 \pm 1.14$ & $46.58 \pm 0.60$ & $56.38 \pm 3.38$ \\
\hline Tetraselmis suecica & $4.27 \pm 0.21$ & $1.71 \pm 0.57$ & $56.46 \pm 3.24$ & $11.35 \pm 0.56$ & $27.38 \pm 1.64$ \\
\hline mean & 2.19 & 2.11 & 25.11 & 30.46 & 23.98 \\
\hline
\end{tabular}

${ }^{\mathrm{a}}$ mean $\pm \operatorname{stdev}(\mathrm{n}=3) ;{ }^{\mathrm{b}}$ n.a. $=$ not available; ${ }^{\mathrm{c}}$ n.d. $=$ not detectable; ${ }^{\mathrm{d}}$ A.C. $=$ mixed culture used in aquaculture $;{ }^{\mathrm{e}}$ W.W.T $=$ mixed culture for waste water treatment 
volume was adjusted to $50 \mathrm{~mL}$ with additional borate buffer, and the emulsion was stored at $4^{\circ} \mathrm{C}$ in the dark under nitrogen until needed. Before use, the substrate was checked for autoxidation, and solutions exhibiting $>3 \%$ autoxidation were discarded. For the assay, $30 \mu \mathrm{L}$ substrate and $10 \mu \mathrm{L}$ antioxidant solution were added to a quartz cuvette containing $2.81 \mathrm{~mL}$ of $0.05 \mathrm{M}$ phosphate buffer $(\mathrm{pH} 7.4)$ at $40^{\circ} \mathrm{C}$. The oxidation reaction was initiated at $37^{\circ} \mathrm{C}$ by adding $150 \mu \mathrm{L}$ of $40 \mathrm{mM}$ AAPH solution freshly prepared in $0.05 \mathrm{M}$ phosphate buffer ( $\mathrm{pH}$ 7.4). For the blank, $10 \mu \mathrm{L}$ of buffer was added instead of antioxidant solution. The radical reactions were monitored by recording the increase in absorption at $234 \mathrm{~nm}$ caused by conjugated diene hydroperoxides. Absorbances were measured at $37^{\circ} \mathrm{C}$ using a Thermo $\mathrm{L}$ spectrophotometer, equipped with a 7-position automatic sample changer, connected to an external water bath. All measurements were run against the phosphate buffer. A separate AAPH-free control was prepared to check for any spontaneous oxidation. The absorbance of an AAPH reference (mixture of $150 \mu \mathrm{L}$ AAPH solution and $2.85 \mathrm{~mL}$ phosphate buffer) was measured in a separate cuvette and the absorbance value is subtracted from each experimental point. (AAPH has a relatively high absorbance below $260 \mathrm{~nm}$, which changes as it decomposes). The inhibition time $\left(\mathrm{T}_{\mathrm{inh}}\right)$ was calculated as the point of intersection between the tangents to the inhibition and propagation phase curves, when plotting the absorbance at $234 \mathrm{~nm}$ versus the reaction time (see Fig. 4).

Phenolic and carotenoid content

Phenolic content was estimated by the Folin-Ciocalteu procedure according to the slightly modified procedure used by Hajimahmoodi et al. (2010). For this, $200 \mu \mathrm{L}$ extract was mixed with $1.5 \mathrm{~mL}$ of Folin-Ciocalteu reagent (previously diluted tenfold with distilled water) and allowed to stand at room temperature for $5 \mathrm{~min}$. Next, $1.5-\mathrm{mL}$ sodium

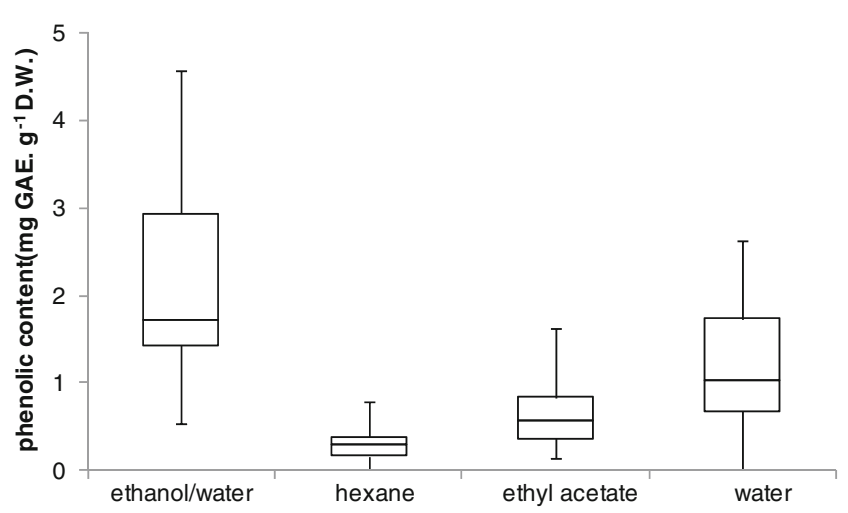

Fig. 2 Box plot distribution of the phenolic content of the microalgal fractions as determined by the Folin-Ciocalteu assay. Error bars represent minimum and maximum values, whereas the rectangle boundaries represent 1 st quartile, median and 3th quartile values bicarbonate solution $\left(60 \mathrm{gL}^{-1}\right)$ was added to the mixture. After incubation for $90 \mathrm{~min}$ at room temperature, the absorbance was measured at $750 \mathrm{~nm}$. Total phenolics were calibrated against gallic acid standard solutions $\left(25-150 \mathrm{mg} \mathrm{L}^{-1}\right.$ in $50 \%$ methanol) and are expressed as $\mathrm{mg}$ gallic acid equivalent (G.A.E.) $\mathrm{g}^{-1}$ biomass.

Carotenoid content was estimated spectrophotometrically according to the method of Lichtenthaler and Buschmann (2001). Aliquots of the extracts were diluted 15-300 times with $90 \%(\mathrm{v} / \mathrm{v})$ methanol in water and absorbances were measured at 470, 652 and $665 \mathrm{~nm}$ and carotenoid content is calculated using the Lichtenthaler equations.
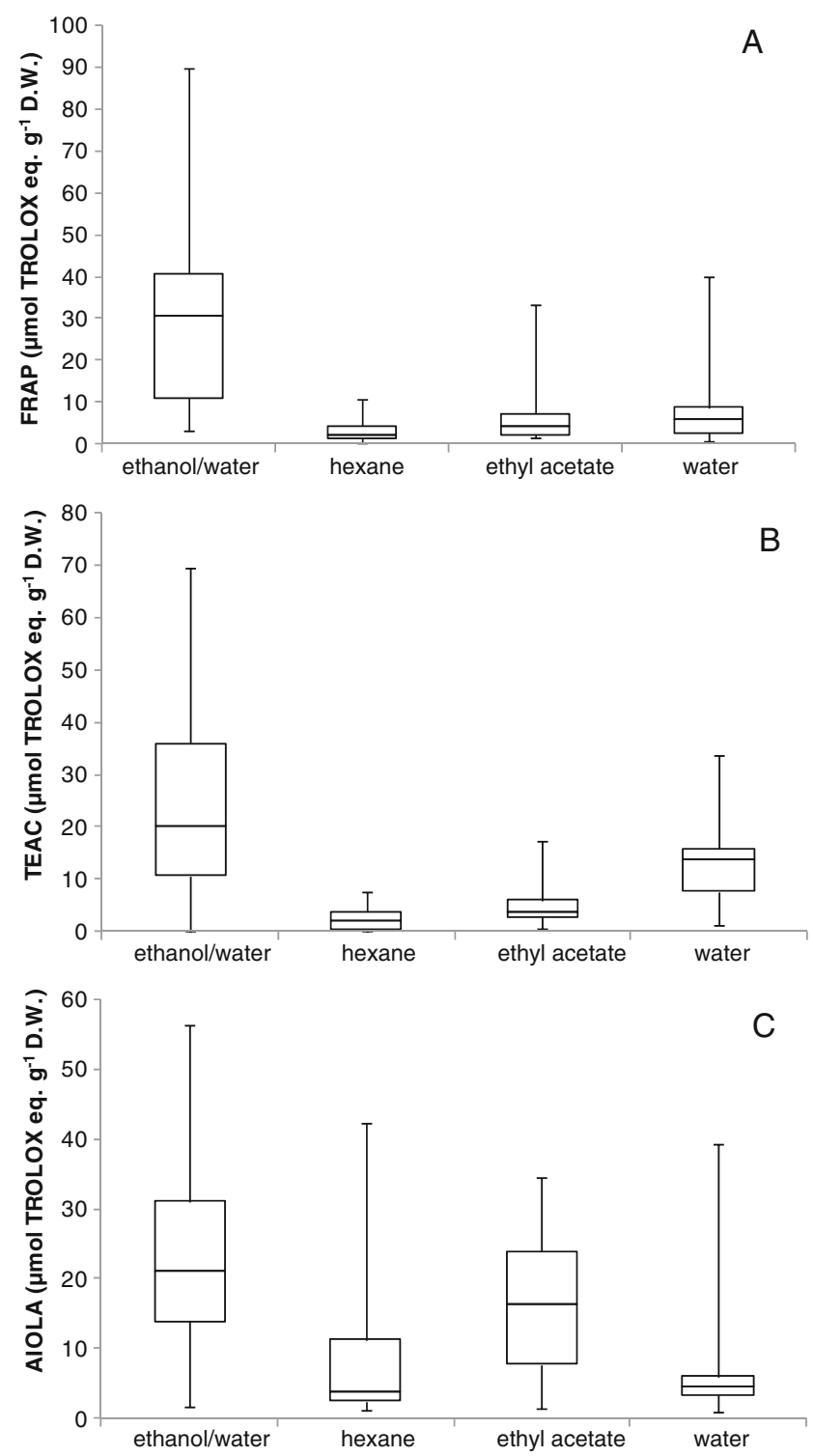

Fig. 3 Box plot distribution of the antioxidant capacity of microalgal fractions as determined by FRAP assay (3A), TEAC assay (3B) and AIOLA assay $(3 \mathrm{C})$. Error bars represent minimum and maximum values, whereas the rectangle boundaries represent $1^{\text {st }}$ quartile, median and $3^{\text {th }}$ quartile values 


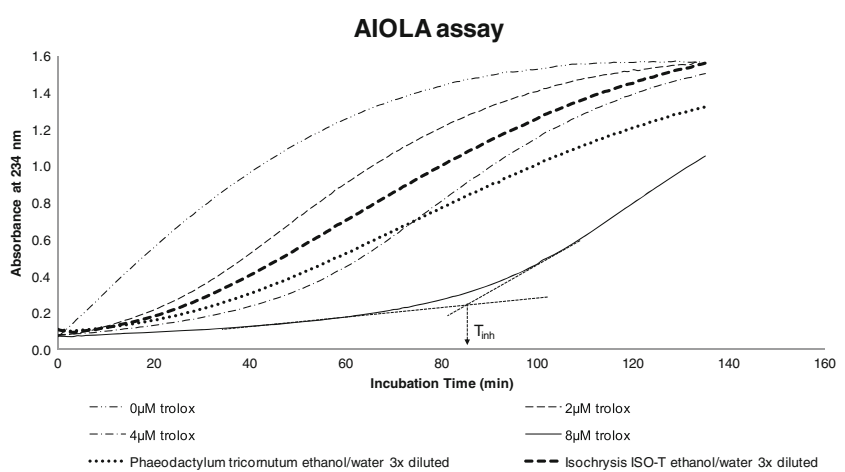

Fig. 4 Typical profile of the formation of conjugated diene hydroperoxides from linoleic acid in the presence of the synthetic vitamin $\mathrm{E}$ analogue trolox or diluted algal extracts. $\mathrm{T}_{\mathrm{inh}}=$ inhibition time

Statistical analyses

The results of three replicate extracts from each sample were used for statistical analysis. Multiple regression analysis was carried out using R-software (version 2.13.0). For the regression model, the antioxidant activity is calculated as a function of both carotenoid and phenolic content. By using a multiple regression model, the contribution of both carotenoid and phenolic content can be determined more accurately than with univariate regression.

\section{Results}

Carotenoid and phenolic content of microalgal biomass

For most species, the highest carotenoid content (Fig. 1) was found in the ethanol/water extracts ranging from 0 to $7.8 \mathrm{mg} \mathrm{g}^{-1}$ biomass (Table 1). Samples with a relatively high carotenoid content ( $>3 \mathrm{mg} \mathrm{g}^{-1}$ biomass) belonged to Isochrysis, Phaeodactylum, Chlorella sp. and T. suecica. The heterotrophically grown Schizochytrium did not contain any carotenoids. In the extracts, obtained by the fractionating procedure, most carotenoids were found in the ethyl acetate fraction, followed by the hexane fraction. The hot water fraction contained only traces of carotenoids. Exceptions were Isochrysis and Haematococcus (green phase), for which most carotenoids were found in the hexane fraction. The observation that carotenoids were not detected in the H. pluvialis biomass (red phase) can be explained by the fact that in this growth phase, the carotenoid fraction consists mainly of astaxanthin (mainly esterified), which apparently is hardly or not extracted with the used ethanol/water mixture.

Phenolic content in the ethanol/water extracts varied from 0.5 to $4.6 \mathrm{mg}$ G.A.E. $\mathrm{g}^{-1}$ biomass (Table 1). Samples with a relatively high phenolic content $\left(>3\right.$ mg G.A.E. $\mathrm{g}^{-1}$ biomass) belonged to Isochrysis, Phaeodactylum, Tetraselmis sp. and a mixed culture of marine diatoms. In contrast to the carotenoids, the highest phenolic content was measured in the hot water fractions, followed by the ethyl acetate fractions (Fig. 2). The hexane fractions showed only a low phenolic content, which is to be expected since phenolic substances are typically more polar compounds.

\section{Antioxidant activity of microalgal biomass}

For the FRAP assay, antioxidant activity in the ethanol/ water extracts varied from 3.3 to $90 \mu \mathrm{mol}$ trolox eq. $\mathrm{g}^{-1}$ biomass (Table 1). Samples with a relatively high FRAP activity ( $>40 \mu$ mol trolox eq. $\mathrm{g}^{-1}$ biomass) belonged to $C$. vulgaris, $H$. pluvialis (green phase), Isochrysis ISO-T and Isochrysis sp., N. oculata, Nannochloropsis sp., P. tricornutum and Tetraselmis sp.. FRAP activity was highest in the hot water fractions and lowest in the hexane fractions for most samples (Fig. 3a). Our results are in agreement with Hajimahmoodi et al. (2010), who measured similar FRAPvalues and who also measured the highest FRAP activity in the hot water fractions when compared to hexane fractions.

Antioxidant activity tested according to the TEAC assay varied from 0 to $69 \mu \mathrm{mol}$ trolox eq. $\mathrm{g}^{-1}$ biomass in ethanol/ water extracts (Table 1). Samples with a relatively high TEAC activity ( $>40 \mu \mathrm{mol}$ trolox eq. $\mathrm{g}^{-1}$ biomass) belonged to B.s braunii, Chlorella sp., N. oleoabundans, P. tricornutum, Tetraselmis sp. and T. suecica. TEAC activity was highest in the hot water fractions and lowest in the hexane fractions (Fig. 3b). Li et al. (2007) also measured TEAC activity in extracts from microalgae. They measured similar TEAC activities and also found the highest TEAC activity in hot water extracts and the lowest activity in hexane extracts.
Table 2 Multiple regression analysis of both phenolic and carotenoid content versus antioxidant activity using t-test for significance

\begin{tabular}{lcccccc}
\hline X-variable & Y-variable & Coefficients & Standard Error & $\mathrm{R}^{2}$ & $\mathrm{t}$ Stat & P-value \\
\hline phenolic content & FRAP value & 8.103 & 1.608 & 0.549 & 5.040 & $2.39 \mathrm{E}-6$ \\
carotenoid content & & 5.553 & 1.403 & & 3.957 & $1.51 \mathrm{E}-4$ \\
phenolic content & TEAC value & 6.464 & 1.501 & 0.510 & 4.306 & $4.83 \mathrm{E}-5$ \\
carotenoid content & & 5.300 & 1.310 & & 4.045 & $1.10 \mathrm{E}-4$ \\
phenolic content & AIOLA value & 3.525 & 1.950 & 0.302 & 1.905 & $6.10 \mathrm{E}-2$ \\
carotenoid content & & 4.358 & 1.529 & & 2.850 & $5.80 \mathrm{E}-3$ \\
\hline
\end{tabular}


Antioxidant activity according to AIOLA assay was only tested on 19 of the 32 biomass samples, and varied from 1.8 to $56 \mu \mathrm{mol}$ trolox eq. $\mathrm{g}^{-1}$ biomass in the ethanol/water extracts (Table 1). Samples with a relatively high AIOLA activity ( $>40 \mu \mathrm{mol}$ trolox eq. $\mathrm{g}^{-1}$ biomass) belonged to samples of $P$. tricornutum, Tetraselmis sp. and a mixture of marine microalgae. In contrast to TEAC and FRAP, AIOLA activity was highest in the order ethyl acetate > hexane $>$ hot water fractions (Fig. 3c). The formation of conjugated diene hydroperoxides in the AIOLA assay is illustrated in Fig. 4. In the absence of antioxidants $(0 \mu \mathrm{M}$ trolox), conjugated diene hydroperoxides are formed immediately after the addition of the radical initiator, whereas the reaction shows a delayed onset in the presence of antioxidants. In a model solution containing the reference antioxidant trolox, the onset of diene hydroperoxide formation is delayed but, once the reaction has been initiated, the rate at which diene hydroperoxides are formed is similar as in the absence of antioxidants. In the presence of algal extracts, on the contrary, the rate at which hydroperoxides are formed after a certain inhibition period is clearly lower, as indicated by a lower slope of the curve compared to the trolox curves. While trolox blocks the oxidative reaction almost completely until all antioxidant has been used up, algal extracts seem to act more as retardant antioxidants as described by Laguerre et al.(2007). This is very likely due to the fact that algal extracts contain a complex mixture of antioxidants in which a specific antioxidant can be regenerated by another antioxidant.

Multiple regression analysis (Table 2) indicated that both carotenoid and phenolic content significantly contributed to explaining the variation in FRAP and TEAC activity of the extracts. The regression coefficients indicate that phenolics and carotenoids are of similar importance in explaining variation in the antioxidant activity. For AIOLA, only the carotenoid content significantly contributed to explaining variation in antioxidant activity in the extracts (Table 2). The contribution of the phenolic content was only marginally significant $(p=0.06)$. The $\mathrm{R}^{2}$ value of the multiple regressions for FRAP (0.549), TEAC (0.510) and AIOLA (0.310) suggest that, besides phenolics and carotenoids, also other components contributed to the antioxidant activities measured in the samples.

\section{Batch-to-batch variations}

Table 1 shows that phenolic content was comparable in different batches of $P$. tricornutum cultured in our pilot-scale photobioreactors. The same holds true for the different $C$. vulgaris batches, with the exception of batch 2 which contained only half of the phenolic content of the other batches. Carotenoid content was also more or less comparable among the 3 Phaeodactylum batches but differed largely between the 6 different Chlorella batches. Samples of
Chlorella and Phaeodactylum biomass supplied by commercial producers had a comparable phenolic content but had a higher carotenoid content than most biomass samples obtained with our pilot installation. The variation in the content of carotenoids and phenolics was reflected in differences in the antioxidant capacity, as measured by the TEAC- and FRAP-assays, which also varied from batch to batch and was higher in the commercial biomass.

\section{Discussion}

It is well-known that carotenoids are important contributors to antioxidant activity in microalgal biomass (see e.g. Kobayashi et al. 1997; Herrero et al. 2006). This was confirmed by our multiple regression analyses, which indicated a significant contribution of carotenoids to antioxidant activity as measured by the FRAP, TEAC and AIOLA assays. However, the fact that antioxidant activity measured according to TEAC and FRAP was highest in the hot water extract fractions while carotenoid content was very low in these fractions implies that carotenoids are not the only determinants of antioxidant activity in microalgae. Indeed, the hot water extracts had the highest content of phenolic compounds and multiple regression analyses indicated that phenolic compounds also contributed significantly to explaining antioxidant activity as measured by the TEAC and FRAP assays.

Comparing the data on carotenoid content with other studies must be made with caution since carotenoid content and composition are influenced by culture conditions such as nutrient availability and light intensity. Nevertheless, the carotenoid content found in the used biomass samples falls within the range given by Spolaore et al. (2006), who noted an average carotenoid content of 1.0 to $2.0 \mathrm{mg} \mathrm{g}^{-1}$ biomass with values up to $140 \mathrm{mg} \mathrm{g}^{-1}$ biomass in D. salina.

Compared to carotenoids, few studies have measured phenolic content in microalgae. Phenolic content as measured in this study was comparable to the previous studies of Geetha et al. (2010), Hajimahmoodi et al. (2010) and Li et al. (2007), although Cha et al. (2010) detected higher levels in extracts from $C$. vulgaris obtained by pressurised liquid extraction at elevated temperatures using $90 \%$ ethanol as extractant. In our study, the highest phenolic content was measured in the hot water fractions, followed by the ethyl acetate fractions and hexane fractions. This can be ascribed to the generally polar nature of phenolic compounds and is in agreement with Hajimahmoodi et al. (2010), who also found the highest phenolic content in the hot water fraction. Surprisingly, however, Li et al. (2007) found the highest content of phenolic substances in the apolar hexane fraction.

Cai et al. (2004) studied the antioxidant activity (using the TEAC assay) and phenolic content of methanol/water 
extracts from a large number of medicinal plants as well as several common edible fruits and vegetables. Mean values for antioxidant activity were $9 \mu \mathrm{mol}$ trolox eq. $\mathrm{g}^{-1}$ biomass for medicinal plants and $0.9 \mu \mathrm{mol}$ trolox eq. $\mathrm{g}^{-1}$ biomass for edible fruits and vegetables. Samples having a TEAC value above $10 \mu \mathrm{mol}$ trolox eq. $\mathrm{g}^{-1}$ biomass were considered to be rich in antioxidants. The mean value for TEAC in our microalgal ethanol/water extracts of $25 \mu \mathrm{mol}$ trolox eq. $\mathrm{g}^{-1}$ biomass and the highest value of $69 \mu \mathrm{mol}$ trolox eq. $\mathrm{g}^{-1}$ biomass (for Tetraselmis sp.) clearly endorse the antioxidant potential of microalgae.

In our study, carotenoid content contributed significantly to explaining TEAC, FRAP and AIOLA activity, whereas phenolic compounds only contributed to explaining TEAC and FRAP activity. Carotenoids are well-known for their ability to deactivate singlet oxygen by physical quenching. Further, carotenoids can quench radicals by hydrogen atom transfer or by accepting electrons from radicals (Martínez and Barbosa 2008). Polyphenols act as antioxidants through single electron transfer and through hydrogen atom transfer. The TEAC assay quantifies antioxidant activity by single electron transfer (direct reduction of $\mathrm{ABTS}^{\bullet+}$ ) or radical quenching by hydrogen atom transfer (Prior et al. 2005), and thus detects antioxidant activity from both carotenoids and polyphenols. The FRAP assay quantifies antioxidant compounds that have redox potentials below $0.7 \mathrm{~V}$ (the redox potential of the $\mathrm{Fe}^{3+}$-TPTZ complex). FRAP detects antioxidants that act through single electron transfer but cannot detect compounds that act as radical quenchers by hydrogen atom transfer (Prior et al. 2005), and should thus mainly detect antioxidant activity from polyphenols. However, recently, Müller et al. (2011) demonstrated that specific carotenes and xanthophylls show significant FRAP activity. The AIOLA assay estimates the ability of compounds to prevent oxidation of lipids in emulsions. The highly conjugated system in carotenoid molecules is very efficient in scavenging oxygen radicals and thus in preventing oxidation of polyunsatured fatty acids. Moreover, in oil-in-water emulsions, the relatively apolar carotenoids can dissolve in the oil phase to prevent lipid oxidation. The fact that the more polar phenolic compounds dissolve to a lesser extent in the oil phase probably explains why they are a poor predictor of AIOLA activity (cf. Shahidi and Zhong 2011). As mentioned above, our AIOLA results only reflect the use of microalgal extracts to prevent lipid oxidation in emulsion, which is apparent for alcohol/water extracts of P. tricornutum, Tetraselmis sp. and a mixture of marine microalgae. These extracts contain potent antioxidants that largely inhibit lipid oxidation under the conditions used.

Differences in antioxidant activity in specific tests may not only be related to the quantity of carotenoids and polyphenols, but also the specific characteristics of carotenoids and polyphenolic identity of the specific compounds. Müller et al. (2011) demonstrated that activity of carotenoids in TEAC and FRAP assays are not only determined by the number of conjugated double bonds, but also by the presence of a hydroxyl group near the conjugated system and the substitution pattern of the $\beta$-ionone rings. These authors described large variation in the reactivity of the different types of carotenoids towards both FRAP and TEAC assays, with carotenes being more active than xanthophylls in the TEAC assay and the opposite for the FRAP assay. It is also well-known that the antioxidant activity of polyphenols, as measured by FRAP (Pulido et al. 2000) or TEAC (RiceEvans et al. 1996), is related to the degree and pattern of hydroxylation and extent of conjugation in polyphenols. From our data, it can be seen that many of the Chlorophyta, such as B. braunii, Chlorella sp., N. oleoabundans and Tetraselmis sp. have high FRAP and TEAC activities. This could be related to relatively high contents in $\beta$-carotene (not active towards FRAP reagent), lutein, violaxanthin and neoxanthin that are found in these microalgae (Takaichi 2011), considering their high reactivity as stated by Müller et al. (2011). The commercial samples of P. tricornutum and Isochrysis may be rich in fucoxanthin, a carotenoid known for its antioxidant power (Sachindra et al. 2007) and typical for Bacillariophyceae and Haptophyta (Takaichi. 2011). Since the multiple regression analysis clearly indicated that both phenolics and carotenoids contribute to FRAP and TEAC antioxidant activities, interpretation of the data on antioxidant activity cannot rely solely on carotenoid composition and information on phenolic content and phenolic composition must be taken into account. At present, little is known about the polyphenolic components that are present in microalgae. Recent work by Klejdus et al. (2010) and Kovácik et al. (2010) indicate that a variety of phenolic classes are present in microalgae, but further identification of phenolic components is needed to better understand the differences in the contribution of phenolics to the antioxidant activity of microalgae.

In conclusion, our results suggest that when using microalgae biomass as a source of natural antioxidants, not only carotenoids but also phenolic compounds should be considered. Although the content of phenolic substances in microalgae is in the lower end of the range reported for terrestrial plants (Cai et al. 2004), it was comparable to the carotenoid content in the studied samples. Further identification of phenolic substances from microalgae is required to evaluate whether microalgae may contain novel phenolic compounds that are not known from terrestrial plants. Further, it should be noted that carotenoid content in some microalgae can be increased up to $140 \mathrm{mg} \mathrm{g}^{-1}$ biomass by manipulating environmental conditions (Spolaore et al. 2006) and that the same might be true for phenolic compounds. The fact that the phenolic content differed almost threefold between different samples of the same species suggests that the phenolic 
content in microalgae may be optimised by selecting the appropriate cultivation and processing conditions.

Acknowledgments This study was funded by the Industrial Research Fund of K.U.Leuven (project number IOF-KPH/09/002) and the Fund for Scientific Research (project G.A129.11 N). The authors wish to thank Maris projects, Necton S.A. and SBAE industries for providing algal biomass samples. Colette Cooreman and Bart Dewaele are acknowledged for their technical assistance. I. Fraeye is a Postdoctoral Researcher funded by the Research Foundation Flanders (FWO).

\section{References}

Cai Y, Luo Q, Sun M, Corke H (2004) Antioxidant activity and phenolic compounds of 112 traditional Chinese medicinal plants associated with anticancer. Life Sci 74:2157-2184

Cerón MC, García-Malea MC, Rivas J, Acien FG, Fernandez JM, Del Río E, Guerrero MG, Molina E (2007) Antioxidant activity of Haematococcus pluvialis cells grown in continuous culture as a function of their carotenoid and fatty acid content. Appl Microbiol Biot 74:1112-1119

Cha KH, Kang SW, Kim CY, Um BH, Na YR, Pan C-H (2010) Effect of pressurized liquids on extraction of antioxidants from Chlorella vulgaris. J Agric Food Chem 58:4756-4761

Chacón-Lee TL, González-Mariño GE (2010) Microalgae for "healthy" foods-possibilities and challenges. CRFSFS 9(6):655675

Custódio L, Justo T, Silvestre L, Barradas A, Duarte CV, Pereira H, Barreira L, Rauter AP, Alberício F, Varela J (2011) Microalgae of different phyla display antioxidant, metal chelating and acetylcholinesterase inhibitory activities. Food Chem. doi:10.1016/j. foodchem.2011.08.047

Cuvelier M-E (2001) Antioxidants. In: Morais R (ed) Functional Foods: An introductory course. Escola Superior de Biotecnologia/UCP, Porto, Portugal, pp 97-108

Duval B, Shetty K, Thomas WH (2000) Phenolic compounds and antioxidant properties in the snow alga Chlamydomonas nivalis after exposure to UV light. J Appl Phycol 11:559-566

Geetha BV, Navasakthi R, Padmini E (2010) Investigation of antioxidant capacity and phytochemical composition of Sun Chlorella an in vitro study. J Aquac Res Development 1:104. doi:10.4172/ 2155-9546.1000104

Goh S-H, Yusoff FM, Loh S-P (2010) A comparison of the antioxidant properties and total phenolic content in a diatom, Chaetoceros sp. and a green microalga, Nannochloropsis sp. J Agr Sci 2:123-130

Guillard RRL, Lorenzen CJ (1972) Yellow-green algae with chlorophyllide c. J Phycol 8:10-14

Guzman S, Gato A, Galleja JM (2001) Antiinflammatory, analgesic and free radical scavenging activities of the marine microalgae Chlorella stigmatophora and Phaeodactylum tricornutum. Phytother Res 15:224-230

Hajimahmoodi M, Faramarzi MA, Mohammadi N, Soltani N, Oveisi MR, Nafissi-Varcheh N (2010) Evaluation of antioxidant properties and total phenolic contents of some strains of microalgae. J Appl Phycol 22:43-50

Herrero M, Jaime L, Martín-Alvarez PJ, Cifuentes A, Ibáñez E (2006) Optimization of the extraction of antioxidants from Dunaliella salina microalga by pressurized liquids. J Agric Food Chem 54:5597-5603

Jahnke L (1999) Massive carotenoid accumulation in Dunaliella bardawil induced by ultraviolet-A radiation. J Photoch Photobiol 48:68-74
Jaime L, Mendiola JA, Herrero M, Soler-Rivas C, Santoyo S, Señorans FJ, Cifuentes A, Ibáñez E (2005) Separation and characterization of antioxidants from Spirulina platensis microalga combining pressurized liquid extraction, TLC, and HPLC-DAD. J Sep Sci 28:2111-2119

Klejdus B, Lojková L, Plaza M, Snóblová M, Stěrbová D (2010) Hyphenated technique for the extraction and determination of isoflavones in algae: Ultrasound-assisted supercritical fluid extraction followed by fast chromatography with tandem mass spectrometry. J Chromatogr A 1217:7956-7965

Kobayashi M, Kakizono T, Nishio N, Nagai S, Kurimura Y, Tsuji Y (1997) Antioxidant role of astaxanthin in the green alga Haematococcus pluvialis. Appl Microbiol Biot 48:351-356

Kovácik J, Klejdus B, Backor M (2010) Physiological responses of Scenedesmus quadricauda (Chlorophyceae) to UV-A and UV-C light. Photochem Photobiol 86:612-616

Laguerre M, Lecomte J, Villeneuve P (2007) Evaluation of the ability of antioxidants to counteract lipid oxidation: Existing methods, new trends and challenges. Prog Lipid Res 46:244-282

Lee S-H, Lee J-B, Lee K-W, Jeon Y-J (2010) Antioxidant properties of tidal pool microalgae, Halochlorococcum porphyrae and Oltamannsiellopsis unicellularis from Jeju Island, Korea. Algae 25:45-56

Li H, Cheng K, Wong C, Fan K, Chen F, Jiang Y (2007) Evaluation of antioxidant capacity and total phenolic content of different fractions of selected microalgae. Food Chem 102:771-776

Lichtenthaler HK, Buschmann C (2001) Chlorophylls and carotenoids: measurement and characterization by UV-VIS. In: Wrolstad RE (ed) Current protocols in food analytical chemistry. John Wiley and Sons, New York, pp F.4.3.1-F.4.3.8

Liégois C, Lermusieau G, Collin S (2000) Measuring antioxidant efficiency of wort, malt and hops against oxidation of an aqueous dispersion of linoleic acid. J Agric Food Chem 48:1129 1134

Martínez A, Barbosa A (2008) Antiradical power of carotenoids and vitamin E: testing the hydrogen atom transfer mechanism. J Phys Chem B 112:16945-16951

Mendiola J, Jaime L, Santoyo S, Reglero G, Cifuentes A, Ibanez E et al (2007) Screening of functional compounds in supercritical fluid extracts from Spirulina platensis. Food Chem 102:1357-1367

Miranda MS, Cintra RG, Barros SB, Mancini Filho J (1998) Antioxidant activity of the microalga Spirulina maxima. Braz J Med Biol Res 31:1075-1079

Müller L, Fröhlich K, Böhm V (2011) Comparative antioxidant activities of carotenoids measured by ferric reducing antioxidant power (FRAP), ABTS bleaching assay ( $\alpha$ TEAC), DPPH assay and peroxyl radical scavenging assay. Food Chem 129:139-148

Namiki M (1990) Antioxidants/antimutagens in food. Crit Rev Food Sci 29:273-300

Natrah FMI, Yusoff FM, Shariff M, Abas F, Mariana NS (2007) Screening of Malaysian indigenous microalgae for antioxidant properties and nutritional value. J Appl Phycol 19:711-718

Pietta PG (2000) Flavonoids as antioxidants. J Nat Prod 63:1035-1042

Pokorný J (1991) Natural antioxidants for food use. Trends Food Sci Tech 2:223-227

Prior RL, Wu X, Schaich K (2005) Standardized methods for the determination of antioxidant capacity and phenolics in foods and dietary supplements. J Agric Food Chem 53:4290-4302

Pulido R, Bravo L, Saura-Calixto F (2000) Antioxidant activity of dietary polyphenols as determined by a modified ferric reducing/ antioxidant power assay. J Agric Food Chem 48:3396-3402

Pulz O, Gross W (2004) Valuable products from biotechnology of microalgae. Appl Microbiol Biot 65:635-648

Rao AR, Sarada R, Baskaran V, Ravishankar GA (2006) Antioxidant activity of Botryococcus braunii extract elucidated in vitro models. J Agric Food Chem 54:4593-4599 
Rice-Evans CA, Miller NJ, Paganga G (1996) Structure-activity relationships of flavonoids and phenolic acids. Free Rad Biol Med 20:933-956

Rodriguez-Garcia I, Guil-Guerrero J (2008) Evaluation of the antioxidant activity of three microalgal species for use as dietary supplements and in the preservation of foods. Food Chem 108:1023-1026

Sachindra N, Sat P, Maed H, Hosokawa M, Niwano Y, Kohno M, Miyashita K (2007) Radical scavenging and singlet oxygen quenching activity of marine carotenoid fucoxanthin and its metabolites. J Agric Food Chem 55:8516-8522
Shahidi F, Zhong Y (2011) Revisiting the polar paradox theory: a critical overview. J Agric Food Chem 59:3499-3504

Spolaore P, Joannis-Cassan C, Duran E, Isambert A (2006) Commercial applications of microalgae. J Biosci Bioeng 101:8796

Takaichi S (2011) Carotenoids in algae: distributions, biosyntheses and functions. Mar Drugs 9:1101-1118

Wu L-C, Ho J-A A, Shieh M-C, Lu I-W (2005) Antioxidant and antiproliferative activities of Spirulina and Chlorella water extracts. J Agric Food Chem 53:4207-4212 